\title{
Role of microRNA-296-3p in the malignant transformation of sinonasal inverted papilloma
}

\author{
TOMOHIKO KAKIZAKI*, HIROMITSU HATAKEYAMA*, YUJI NAKAMARU, DAI TAKAGI, \\ TAKATSUGU MIZUMACHI, TOMOHIRO SAKASHITA, SATOSHI KANO, \\ AKIHIRO HOMMA and SATOSHI FUKUDA
}

Department of Otolaryngology-Head and Neck Surgery, Hokkaido University Graduate School of Medicine, Sapporo 060-8638, Japan

Received March 24, 2016; Accepted March 3, 2017

DOI: $10.3892 / \mathrm{ol} .2017 .6193$

\begin{abstract}
Inverted papilloma (IP) is a benign tumor occurring in the nasal cavity and paranasal sinuses. It is reported that $5-15 \%$ of IPs undergo malignant transformation into squamous cell carcinoma (SCC), and the role of microRNAs (miRNA/miR) in this process remains to be elucidated. In the present study, whole miRNA profiles using samples of IP and SCC were investigated, in order to detect the function of miRNA in the carcinogenesis of IP. Samples from IPs $(n=5)$ and SCC lesions $(n=5)$, which arose from IPs, were used for miRNA analysis. A total of 200 miRNAs exhibited a $>2$-fold differential expression between IP and SCC. miR-296-3p was markedly upregulated in SCC with a 23 -fold difference. Computational analysis indicated that miR-296-3p targeted PTEN, which regulates the phosphoinositide 3-kinase (PI3K)/protein kinase B (Akt) signaling pathway and PTEN is involved in the carcinogenesis of SCC. miR-296-3p directly regulated PTEN expression in head and neck cancer cells, with PTEN protein levels decreased in 4/19 the SCCs (21.0\%), as compared with those in the IPs (76.4\%). Positive p21 staining was observed in $64.7 \%$ of IPs; this was a significantly increased rate compared with that for SCCs $(26.3 \%, \mathrm{P}=0.0086)$. The results of the present study indicated that there were marked changes in the miRNA expression signature during the malignant transition. miR-296-3p may serve an important role in the malignant transformation of IPs via the regulation of PTEN, combined with the subsequent inhibition of the PI3K/Akt signaling pathway, and may be a novel agent for cancer prevention.
\end{abstract}

Correspondence to: Dr Hiromitsu Hatakeyama, Department of Otolaryngology-Head and Neck Surgery, Hokkaido University Graduate School of Medicine, Kita 15, Nishi 7, Kita-ku, Sapporo 060-8638, Japan

E-mail: htkym@med.hokudai.ac.jp

*Contributed equally

Key words: sinonasal inverted papilloma, microRNA 296-3p, malignant transformation, phosphatase and tensin homolog

\section{Introduction}

Inverted papilloma (IP) is a benign tumor occurring in the nasal cavity and paranasal sinuses (1-3). IPs typically arise from the lateral wall of the nasal cavity and may spread into the surrounding tissue, the ethmoid, the skull base and the orbita (3). Although surgical treatment has developed through the integration of skull base surgery, navigation systems and endoscopic surgical techniques (1), the management of this disease has remained challenging as IPs tend to recur following incomplete resection, and the malignant transformation to squamous cell carcinoma (SCC) occurs in 5-15\% of cases $(2,3)$.

MicroRNAs (miRNA/miR) are small non-coding RNAs of $\sim 19-22$ nucleotides that regulate gene expression through translational repression and mRNA cleavage (4). In total, $>1,500$ types of human miRNA have been detected and registered, with a number of them reported to be associated with carcinogenesis, differentiation, apoptosis and metastasis (5-7). In the head and neck field, miRNAs are aberrantly expressed in SCC tissue and are associated with carcinogenesis and drug-resistance (5-7).

High-risk human papilloma virus (HPV) infection is regarded as a potential etiological agent of IPs and their malignant transformation $(3,8)$. Carcinogenesis resulting from HPV infection has previously been explained by the inhibition of tumor suppressors, including the p53 and retinoblastoma protein (pRB) signaling pathways (9). The E6 and E7 regions of high-risk HPV are transfected into the genes of epithelial basal cells following infection (10). E6 binds to p53 and promotes the ubiquitination and degradation of p53 itself. E7 also suppresses the cell cycle regulators of $\mathrm{p} 21$ and p27, and binds directly to pRB to promote aggressive cell growth (10). However, it is not always possible to detect high-risk HPV in sinonasal SCCs arising from IPs; the detection rate of high-risk HPV is $\sim 37.8 \%$ (10). The rate of unapparent infection with HPV remains unknown, but a considerable number of such cases are speculated to exist (4). The majority of IPs require a lengthy period for malignant transformation, but the trigger underlying the malignant changes remains obscure (10). SCC arising from IP is one of the best models for studies, as certain tissue samples obtained via surgical resection may contain 
normal epithelium, dysplasia and SCC (4). The present study aimed to identify the underlying mechanisms and roles of miRNAs in the malignant transformation of IPs.

\section{Materials and methods}

Samples and cell lines. A total of 36 individual nasal and paranasal tumor specimens from 25 patients $(19$ males and 6 females) were obtained during surgery at Hokkaido University Hospital (Sapporo, Japan) between January 1993 and October 2012. All samples were obtained prior to radiotherapy and chemotherapy. The median age of the patients was 64.7 years (range, 39-93 years) at the time of diagnosis. The details of the pretreatment clinical and pathological characteristics are summarized in Table I. SCC25 cells were purchased from the American Type Culture Collection (Manassas, VA, USA). Written informed consent was obtained from all patients. The present study was approved by the Institutional Ethics Review Board of Hokkaido University Hospital.

RNA isolation and miRNA TaqMan ${ }^{T *}$ low density array (TLDA) assay. Total RNA was isolated from the formalin-fixed tissue using a Recover All Total Nucleic Acid Isolation kit (Ambion; Thermo Fisher Scientific, Inc., Waltham, MA, USA), according to the manufacturer's protocol. Formalin-fixed tumor tissues were cut into 4-um thick sections, deparaffinized in xylene, rehydrated through graded ethanol, and stained with hematoxylin and eosin. These sections were examined for histopathological accuracy under a light microscope. Unstained sections for RNA isolation were cut into 8-um thick sections and macro-dissected to avoid contamination with other tissues. The quality and quantity of the RNA was determined using an Agilent RNA 6000 Nano LabChip kit and an Agilent 2100 Bioanalyzer (Agilent Technologies, Inc., Santa Clara, CA, USA). Total RNA was used to run the ABI Megaplex ${ }^{\mathrm{TM}}$ protocol without pre-amplification (Applied Biosystems; Thermo Fisher Scientific, Inc.). A reaction was run using an ABI miRNA Reverse Transcription (RT) kit (Applied Biosystems; Thermo Fisher Scientific, Inc.) and Megaplex $^{\mathrm{TMT}}$ RT Human Pool A primers (Applied Biosystems; Thermo Fisher Scientific, Inc.). The cDNA from each sample was then transferred to a new tube and diluted with water and TaqMan ${ }^{\text {Tw }}$ Universal polymerase chain reaction (PCR) Master Mix (2X) without uracil-DNA glycosylase (Applied Biosystems; Thermo Fisher Scientific, Inc.). Each sample was then loaded onto its own TLDA card. TLDAs were queued into the ABI7900HT Real Time PCR machine (Applied Biosystems; Thermo Fisher Scientific, Inc.) and run according to the Applied Biosystems default TLDA protocol. Data were normalized based on the endogenous control on the TLDA card and analyzed using DataAssist ${ }^{\mathrm{TM}}$ v3.0 software (Applied Biosystems; Thermo Fisher Scientific, Inc).

Transfection of miRNA. The miRNA mimics/inhibitor and negative controls were purchased from GE Healthcare Dharmacon, Inc. (Lafayette, CO, USA) and introduced into SCC25 cells using Lipofectamine ${ }^{\circledR} 2000$ (Thermo Fisher Scientific, Inc.) at $37^{\circ} \mathrm{C}$ according to the manufacturer's protocol. Following $6 \mathrm{~h}$, the transfection medium was aspirated and Dulbecco's modified Eagle's medium (Sigma-Aldrich; Merck
Table I. Baseline characteristics of patients with IP and SCC.

A, Cases for microRNA analysis

\begin{tabular}{lc}
\hline Characteristics & Patients, $\mathrm{n}$ \\
\hline Total & 8 \\
Sex & 7 \\
Male & 1 \\
Female & \\
Age, years & $38-77(54)$ \\
Range (median) & \\
\hline & \\
B, Cases for immunohistochemistry & 25 \\
\hline Total & 19 \\
Sex & 6 \\
Male & \\
Female & $32-84(63)$ \\
Age, years & \\
Range, median & 6 \\
Histology & 12 \\
IP non-dysplastic & 7 \\
IP coexisting with SCC & \\
SCC & \\
\hline
\end{tabular}

IP, inverted papilloma; SCC, squamous cell carcinoma.

KGaA, Darmstadt, Germany) with $10 \%$ fetal calf serum (Gibco; Thermo Fisher Scientific, Inc.) was added. Cells were lysed $48 \mathrm{~h}$ following transfection as described below. Transfection was confirmed using miRIDIAN miRNA Mimic Transfection Control with Dy547 (GE Healthcare Dharmacon, Inc.).

Immunoblotting. Cells were lysed with radioimmunoprecipitation assay lysis buffer ( $1 \mathrm{mM}$ NaVO3, $1 \mathrm{mM}$ dithioreitol, $1 \mathrm{mM}$ phenylmethylsulfonyl fluoride (Sigma-Aldrich; Merck $\mathrm{KGaA}$ ), phosphatase inhibitor cocktail and protease inhibitor cocktail mini tablet (Roche Diagnostics, Indianapolis, IN, USA) on ice. The samples were centrifuged at 10,000 x $\mathrm{g}$ for $4 \mathrm{~min}$ and the supernatant was collected. Protein concentration was quantified with a standard Bradford absorbance assay according to the manufacturer's protocol (Bio-Rad, Hercules, CA, USA). Protein from each sample was fractionated on a 4-15\% SDS-PAGE gel (Bio-Rad). The proteins were then transferred to nitrocellulose membranes and blocked with skim milk at room temperature for $1 \mathrm{~h}$. Then, membranes were incubated for $12 \mathrm{~h}$ at $4^{\circ} \mathrm{C}$ with the appropriate primary antibodies phosphatase and tensin homolog (PTEN; cat no. 9559; dilution, 1:1,000), p21 (cat no. 2947; dilution, 1:1,000) (both Cell Signaling Technology, Inc., Danvers, MA, USA) and $\beta$-actin (cat no. ab8226; Abcam, Cambridge, UK; dilution, 1:1,000); followed by incubation with secondary anti-rabbit or anti-mouse HRP-linked antibodies (cat nos., 7074 and 7076, respectively; Cell Signaling Technology, Inc., Danvers, MA, 
Table II. Top 10 miRNAs that exhibited differential expression between SCC and IP.

\begin{tabular}{|c|c|c|c|}
\hline \multicolumn{2}{|c|}{ Upregulated in SCC } & \multicolumn{2}{|c|}{ Downregulated in SCC } \\
\hline Name & Fold-change & Name & Fold-change \\
\hline hsa-miR-206 & 37.74 & hsa-miR-892b & 183.83 \\
\hline hsa-miR-675 & 28.9 & hsa-miR-874 & 144.4 \\
\hline hsa-miR-105 & 25.38 & hsa-miR-371-3p & 121.14 \\
\hline hsa-miR-296-3p & 23.92 & hsa-miR-16-2 & 116.27 \\
\hline hsa-miR-577 & 21.88 & hsa-miR-129 & 85.26 \\
\hline hsa-miR-574-3p & 12.94 & hsa-miR-518f & 70.78 \\
\hline hsa-miR-93 & 9.09 & hsa-miR-431 & 59.55 \\
\hline hsa-miR-936 & 8.87 & hsa-miR-593 & 49.27 \\
\hline hsa-miR-922 & 8.75 & hsa-miR-1225-3p & 45.06 \\
\hline hsa-miR-616 & 8.68 & hsa-miR-92b & 43.71 \\
\hline
\end{tabular}

IP, inverted papilloma; SCC, squamous cell carcinoma; miR, microRNA; hsa, Homo sapiens.

USA) at the manufactures' indicated dilution ratio. Protein bands were visualized using ECL Western Blotting Detection reagent (GE Healthcare Life Sciences, Chalfont, UK) Signal intensities were determined using Image Gauge software (version 4.1; Fujifilm, Tokyo, Japan). Each gel was normalized to $\beta$-actin.

Immunohistochemistry. Paraffin-embedded tumor specimens from the primary sites were available from all 25 patients involved. These specimens were cut into 4-um thick sections, deparaffinized in xylene, rehydrated through graded ethanol and then placed in $0.1 \%$ hydrogen peroxide to quench any endogenous peroxidase activity at room temperature for $10 \mathrm{~min}$. Following pretreatment in a $0.01 \mathrm{M}$ citrate buffer $(\mathrm{pH}$ 6.0) for three cycles of $5 \mathrm{~min}$ at $750 \mathrm{~W}$ in a microwave, these sections were treated with $10 \%$ normal rabbit serum (Histofine ${ }^{\circledR}$ SAB-PO M kit; Nichirei Biosciences, Inc., Tokyo, Japan) at room temperature for 10 min to prevent any nonspecific binding of the antibody. The slides were then incubated with a specific monoclonal antibody against human PTEN or p21 (Cell Signaling Technology) in a humid chamber at $4^{\circ} \mathrm{C}$ overnight. The sections were then incubated with a biotin-labeled rabbit anti-mouse secondary antibody according to the manufacturer's protocol (Histofine SAB-PO M kit) for $30 \mathrm{~min}$ at room temperature, followed by incubation with a streptavidin-biotin horseradish peroxidase complex (Histofine SAB-PO M kit). The reaction products were observed by immersing the slides in a freshly prepared diaminobenzidine solution for $10 \mathrm{~min}$ and counterstaining with hematoxylin at room temperature prior to dehydration and mounting. Stained slides were scored based on the staining intensity and the percentage of positive cells. Staining intensity was scored using a 4-point system as previously described (11) $(0+$, negative; $1+$, weak; $2+$, moderate; $3+$, strong). The positive cell percentage was scored using a 5 -point system $(0,0 \% ; 1+, 1-10 \% ; 2+, 11-50 \%$; $3+, 51-80 \% ; 4+,>80 \%)$. PTEN expression was estimated by cytoplasmic staining, and p21 expression was estimated by nuclear staining. The expression of the two proteins was scored semi-quantitatively using the immunoreactive score (IRS), which was calculated as follows: IRS=staining intensity score $\mathrm{x}$ score of percentage of positive cells. Positivity for PTEN and p21 was defined as an IRS score $\geq 3$, according to the Nagata score (10). In all cases, the procedure was performed by two pathologists, who were blinded to the clinical outcomes. Unpaired t-tests were performed using GraphPad Prism software (version 5.0; GraphPad Software, Inc., La Jolla, CA, USA), and $\mathrm{P}<0.05$ was considered to indicate a statistically significant difference.

Biological targets of miRNAs were predicted by searching for the presence of sites that match the seed region of each miRNA using the TargetScan 6.2 database (http://www. targetscan.org).

\section{Results}

Differential miRNA expression between IP and SCC cells. Using a total of 10 samples (IP=5 and $\mathrm{SCC}=5)$, the role of miRNAs in the carcinogenesis of IP was examined. All five IP samples exhibited transformation to SCCs and all five SCC samples had arisen from IPs. A total of two IP and SCC samples were obtained from the same case (Table IA). miRNA expression analyses were performed using RT-PCR-based arrays to examine 754 unique miRNAs. Among the differentially-expressed miRNAs, the 10 that exhibited the greatest differential expression between IP and SCC are presented in Table II. miR-296-3p exhibited a 23-fold decrease in IP, compared with in SCC. miR-296-3p also demonstrated a 76-fold difference in expression between the IP and SCC from the same cases. A list of 66 target genes was obtained using TargetScan 6.2, including 66 conserved sites and 26 poorly conserved sites that are putatively targeted by miR-296-3p. PTEN, which was identified as a tumor suppressor and involved in the p53 and RB pathways, was included in the list of miR-296-3p target genes.

PTEN is a direct target of miR-296-3p in head and neck SCC (HNSCC) cells. Target prediction using TargetScan 6.2 
revealed that miR-296-3p potentially targets PTEN, as seven nucleotides of the 5 ' seed region were complementary to the PTEN 3'UTR (Fig. 1A). It was established that miR-296-3p negatively regulates PTEN by adding a miR-296-3p mimic into SCC25 cells. The HNSCC cell line, SCC25, was transfected with a miR-296-3p mimic, miR-616 mimic, and a negative control. miR-616 mimic, which was upregulated in SCC cells but did not target PTEN or p21 in silico, was used as the negative control. Following transfection with the miR-296-3p, PTEN expression was markedly decreased, compared with that for the negative control (Fig. 1B). p21, a cyclin-dependent kinase inhibitor, is directly regulated by p53 and is involved in the carcinogenesis of HNSCC. p21 was also reported to be a target of miR-296-5p; therefore, p21 expression was investigated in HNSCC cells transfected with miRNA-296-3p. No significant difference was observed between the p21 expression following transfection with the miRNA-296-3p mimic and with the control (Fig. 1B).

Expression of PTEN and 21 in IP and SCC, and its association with clinicopathological features. Immunohistochemical staining for PTEN and p21 was performed for 17 IP and 19 SCC tissue samples. A total of 11 IPs coexisted with severe dysplasia or SCC, while the remaining six samples were non-dysplastic IPs. A total of 12 SCC samples were differentiated from IPs and the other 7 SCC samples did not coexist with IPs. One IP that coexisted with SCC samples was too small and badly damaged and, therefore, it was excluded from the present study. Representative images of staining for PTEN and p21 are presented in Fig. 2. PTEN expression was observed primarily in the cytoplasm of the tumor cells (Fig. 2C and D). Positive PTEN staining was observed in 13/17 IPs (76.4\%), a rate significantly increased compared with that observed in SCCs $(4 / 19,21.0 \%$; $\mathrm{P}=0.0005$; unpaired t-test with Welch's correlation; Table III). Positive p21 staining was observed in 11/17 IPs (64.7\%), which was also a significantly increased rate compared with that in SCCs $(5 / 19$, 26.3\%; $\mathrm{P}=0.0086$; Table III). No significant differences were observed in PTEN or p21 expression between non-dysplastic and dysplastic IPs, or between SCCs with IPs and SCCs without IPs. Double positive staining was observed in 12 of all the tissue samples, and double negative staining was observed in 15 tissue samples.

\section{Discussion}

In the present study, it was revealed that the expression of numerous miRNAs was altered during the transition between sinonasal IP and SCC. In view of the multistep carcinogenetic process, a large number of miRNAs are considered to be associated with the malignant transformation through the regulation of oncogenes (12). In the present study, number of miRNAs were detected that demonstrated differential expression between IP and SCC, but the majority were not expected to regulate the expression of oncogenes in in silico analysis. Regulation of PTEN by miRNA 296-3p may be one of the critical processes associated with multiple molecular alterations for transformation to invasive SCC.

PTEN is a well-known tumor suppressor gene that is associated with human carcinogenesis (13). PTEN transcription
A

\section{Position 3241-3247 of PTEN 3' UTR}

5' ...UAUUGUGUUUGUUAACAACCCUU... | | | | | | CCUCUCGGAGGAGGGUUGGGAG hsa-miR-296-3p

B

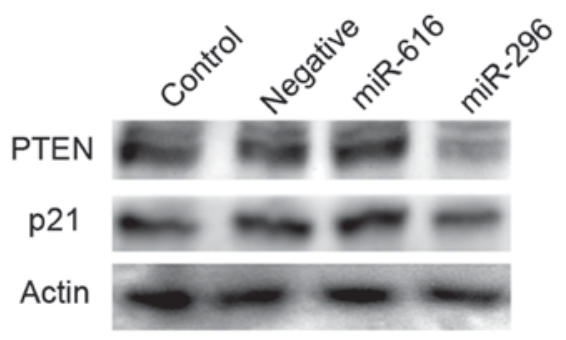

Figure 1. PTEN is a potential target of miR-296-3p. (A) The putative binding site of miR-296-3p in the PTEN 3'UTR site is indicated. (B) The protein levels of PTEN and p21 in SCC25 cells were assessed at $48 \mathrm{~h}$ post-transfection with miR-296-3p mimics, miR-616 and a negative control. PTEN, phosphatase and tensin homolog; UTR, untranslated region; miR, microRNA; hsa, Homo sapiens.

is activated by $\mathrm{p} 53$ and negatively regulates cellular survival by inhibiting the phosphoinositide 3-kinase (PI3K)/protein kinase B (Akt) signaling pathway (13). The PI3K/Akt signaling pathway performs important roles in the carcinogenesis of HNSCC (14). However, a PTEN deficiency of $\leq 30 \%$ is observed in HNSCC (15). Recent genome-wide sequence analysis of HNSCC has revealed a large number of gene alterations, including the molecules involved in the PI3K/Akt signaling pathway $(16,17)$. PTEN mutations were detected in only 5-10\% of cases, and phosphatidylinositol-4,5-bisphosphate 3-kinase catalytic subunit $\alpha$ mutations in $8-10 \%$ of cases $(16,17)$. Therefore, activation of the PI3K/Akt pathway due to PTEN deletion alone is insufficient for the induction of invasive HNSCC. Bian et al (18) promoted HNSCC via the deletion of transforming growth factor- $\beta 1$ and PTEN. Squarize et al (19) demonstrated that mice harboring a PTEN deficiency developed multiple oral SCC lesions on exposure to a tobacco surrogate, whereas the control mice did not.

p21 is a major downstream molecular target of p53 that mediates the apoptotic effect of p53 through the suppression of DNA replication and $\mathrm{G}_{1}$ stage cell cycle arrest (20). The results of the present study demonstrate that the presence of p21 was less common in SCC, as compared with in IP cells, but miRNA-296-3p may not regulate $\mathrm{p} 21$ expression in head and neck cancer cells. In cancer tissue, p21 expression is not always associated with p53 expression as p21 expression levels are low in tissues containing p53 harboring a functional mutation (20). The prognosis and aggressiveness of head and neck cancer were revealed to be associated with the expression of PTEN, but not with p53 and p21 $(21,22)$. In the present study, PTEN and p21 expression were positively correlated with one another, and these results indicate that the trigger for the malignant transformation of IPs to SCCs may underlie the activation of the PI3K/Akt signaling pathway via the loss of PTEN and the subsequent degradation of the p53-p21 pathway.

Several studies have demonstrated the overexpression of miRNA-296 in breast cancer cells (23), glioma cells (24) and 
Table III. The expression status of PTEN and p21.

\begin{tabular}{|c|c|c|c|c|c|c|c|}
\hline Cancer type & Samples, $\mathrm{n}$ & $\begin{array}{c}\text { PTEN-positive, } \\
\mathrm{n}(\%)\end{array}$ & $\begin{array}{c}\text { PTEN-negative, } \\
\mathrm{n}(\%)\end{array}$ & P-value & $\begin{array}{c}\text { p21-positive, } \\
\text { n (\%) }\end{array}$ & $\begin{array}{c}\text { p21-negative, } \\
\text { n (\%) }\end{array}$ & P-value \\
\hline Total & 36 & & & & & & \\
\hline IP non-dysplastic & 6 & 5 & 1 & & 3 & 3 & \\
\hline IP with SCC & 11 & 8 & 3 & & 8 & 3 & \\
\hline & & & & n.s. & & & n.s. \\
\hline SCC arise from IP & 12 & 2 & 10 & & 3 & 9 & \\
\hline SCC & 7 & 2 & 5 & & 2 & 5 & \\
\hline $\begin{array}{l}\text { All IP } \\
\text { All SCC }\end{array}$ & $\begin{array}{l}17 \\
19\end{array}$ & $\begin{array}{c}13(76.5) \\
4(21.1)\end{array}$ & $\begin{array}{c}4(23.5) \\
15(79.9)\end{array}$ & n.s. & $\begin{array}{c}11(64.7) \\
5(26.3)\end{array}$ & $\begin{array}{c}6(35.3) \\
14(73.7)\end{array}$ & n.s. \\
\hline & & & & 0.0005 & & & 0.0086 \\
\hline
\end{tabular}

IP, inverted papilloma; SCC, squamous cell carcinoma; n.s., not significant; PTEN, phosphatase and tensin homolog.
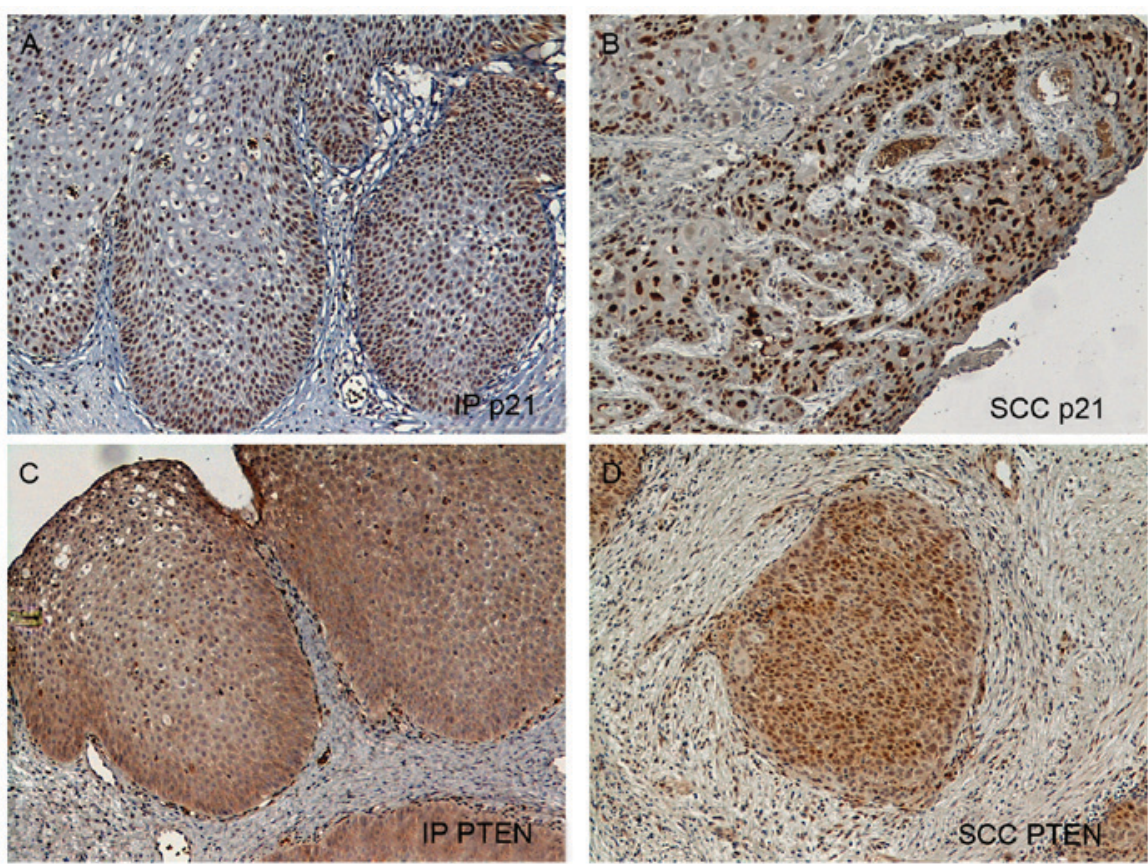

Figure 2. Immunoexpression of PTEN and p21 in IP and SCC. Original magnification, x200. (A) Positive p21 staining in IP. (B) Positive p21 staining in SCC. (C) Positive PTEN staining in IP. (D) Positive PTEN staining in SCC. PTEN, phosphatase and tensin homolog; IP, inverted papilloma; SCC, squamous cell carcinoma.

esophageal cancer (25). In these three studies, miR-296 was used to represent miRNA-296-5p+ (miRNA-296 from the 5' end), and its target genes were distinctly different from the targets of miRNA-296-3p. A previous study determined that miRNA-296-3p elevates and regulates intercellular adhesion molecule 1 , in order to promote prostate cancer metastasis by possibly enhancing the survival of natural killer cell-resistant circulating tumor cells (26). In silico analysis has demonstrated miR-296-3p has 66 target genes, including carcinogenesis related-gene as zinc finger protein 629 and claudin-2. These genes may be involved in the attainment of invasion and metastases in the malignant transformation of IP. The present study focused on miR-296-3p, but the expression of 200 miRNAs were revealed to vary by $>2$-fold during malignant transformation. Each miRNA exhibiting differential expression may perform a role in carcinogenesis; however, not all miRNAs are hypothesized to be associated with it. To identify the function of miRNAs in carcinogenesis, further comprehensive biological molecular studies are required. Recent developments in omics research as an analysis tool for the transcriptome and proteome have made it possible to identify cause and effect associations $(27,28)$. Furthermore, exome studies using next-generation sequencers have been developed $(15,16)$, and the association between genetic alterations and miRNA expression may now be investigated (16). 
In conclusion, the present results indicate that miRNA expression varies during the malignant transformation of sinonasal IPs, and that miR-296-3p, which is overexpressed in SCCs, regulates PTEN expression. The findings of the present study have significant implications regarding the development of carcinogenesis biomarkers, as well as preventative and therapeutic strategies for SCCs arising from IP via the targeting of miRNAs, and the PI3K/Akt and p53-p21 signaling pathways.

\section{Acknowledgements}

The present study was supported by a KAKENHI Grant-in-Aid for Scientific Research from the Ministry of Education, Science and Culture (grant no. 26462591), a Health and Labor Sciences Research Grant for Clinical Cancer Research from the Ministry of Health, Labor and Welfare of Japan (grant no. H26-141) and the National Cancer Center Research and Development Fund of Japan (grant no. 26-A-4).

\section{References}

1. Nakamaru Y, Furuta Y, Takagi D, Oridate N and Fukuda S: Preservation of the nasolacrimal duct during endoscopic medial maxillectomy for sinonasal inverted papilloma. Rhinology 48: 452-456, 2010

2. Myers EN, Fernau JL, Johnson JT, Tabet JC and Barnes EL: Management of inverted papilloma. Laryngoscope 100: 481-490, 1990.

3. Furuta Y, Shinohara T, Sano K, Nagashima K, Inoue K, Tanaka K and Inuyama Y: Molecular pathologic study of human papillomavirus infection in inverted papilloma and squamous cell carcinoma of the nasal cavities and paranasal sinuses. Laryngoscope 101: 79-85, 1991.

4. Bartel DP: MicroRNAs: Target recognition and regulatory functions. Cell 136: 215-233, 2009.

5. Kinoshita T, Hanazawa T, Nohata N, Okamoto Y and Seki N: The functional significance of microRNA-375 in human squamous cell carcinoma: Aberrant expression and effects on cancer pathways. J Hum Genet 57: 556-563, 2012.

6. Christensen BC, Moyer BJ, Avissar M, Ouellet LG, Plaza SL, McClean MD, Marsit CJ and Kelsey KT: A let-7 microRNA-binding site polymorphism in the KRAS 3'UTR is associated with reduced survival in oral cancers. Carcinogenesis 30: 1003-1007, 2009.

7. Hatakeyama H, Cheng H, Wirth P, Counsell A, Marcrom SR, Wood CB,Pohlmann PR, Gilbert J, Murphy B, Yarbrough WG, et al: Regulation of heparin-binding EGF-like growth factor by miR-212 and acquired cetuximab-resistance in head and neck squamous cell carcinoma. PLoS One 5: e12702, 2011.

8. Syrjänen S: Human papillomavirus (HPV) in head and neck cancer. J Clin Virol 32 (Suppl 1): S59-S66, 2005.

9. zur Hausen H: Papillomaviruses causing cancer: Evasion from host-cell control in early events in carcinogenesis. J Natl Cancer Inst 92: 690-698, 2000.

10. Syrjänen K and Syrjanen S: Detection of human papillomavirus in sinonasal carcinoma: Systematic review and meta-analysis. Hum Pathol 44: 983-991, 2013.

11. Nagata Y,Lan KH,Zhou X, Tan M, Esteva FJ, Sahin AA, Klos KS, Li P, Monia BP, Nguyen NT, et al: PTEN activation contributes to tumor inhibition by trastuzumab and loss of PTEN predicts trastuzumab resistance in patients. Cancer Cell 6: 117-127, 2004.

12. Courthod G, Franco P, Palermo L, Pisconti S and Numico G: The role of microRNA in head and neck cancer: Current knowledge and perspectives. Molecules 19: 5704-5716, 2014.
13. Stambolic V, MacPherson D, Sas D, Lin Y, Snow B, Jang Y, Benchimol S and Mak TW: Regulation of PTEN transcription by p53. Mol Cell 8: 317-325, 2001.

14. Pedrero JM, Carracedo DG, Pinto CM, Zapatero AH, Rodrigo JP, Nieto CS and Gonzalez MV: Frequent genetic and biochemical alterations of the PI 3-K/AKT/PTEN pathway in head and neck squamous cell carcinoma. Int J Cancer 114: 242-248, 2005.

15. da Costa AA, D'Almeida Costa F, Ribeiro AR, Guimarães AP, Chinen LT, Lopes CA and de Lima VC: Low PTEN expression is associated with worse overall survival in head and neck squamous cell carcinoma patients treated with chemotherapy and cetuximab. Int J Clin Oncol 20: 282-289, 2015.

16. Stransky N, Egloff AM, Tward AD, Kostic AD, Cibulskis K, Sivachenko A, Kryukov GV, Lawrence MS, Sougnez C, McKenna A, et al: The mutational landscape of head and neck squamous cell carcinoma. Science 333: 1157-1160, 2011.

17. Agrawal N, Frederick MJ, Pickering CR, Bettegowda C, Chang K, Li RJ, Fakhry C, Xie TX, Zhang J, Wang J, et al: Exome sequencing of head and neck squamous cell carcinoma reveals inactivating mutations in NOTCH1. Science 333: 1154-1157, 2011.

18. Bian Y, Hall B, Sun ZJ, Molinolo A, Chen W, Gutkind JS, Waes CV and Kulkarni AB: Loss of TGF- $\beta$ signaling and PTEN promotes head and neck squamous cell carcinoma through cellular senescence evasion and cancer-related inflammation. Oncogene 31: 3322-3332, 2012.

19. Squarize CH, Castilho RM, Abrahao AC, Molinolo A, Lingen MW and Gutkind JS: PTEN deficiency contributes to the development and progression of head and neck cancer. Neoplasia 15: 461-471, 2013.

20. Schwerer MJ, Sailer A, Kraft K, Baczako K and Maier H: Patterns of p21 (waf1/cip1) expression in non-papillomatous nasal mucosa, endophytic sinonasal papillomas and associated carcinomas. J Clin Pathol 54: 871-876, 2001.

21. Sham CL, To KF, Chan PK, Lee DL, Tong MC and van Hasselt CA: Prevalence of human papillomavirus, Epstein-Barr virus, p21, and p53 expression in sinonasal inverted papilloma, nasal polyp, and hypertrophied turbinate in Hong Kong patients. Head Neck 34: 520-533, 2012.

22. Kim SG, Lee OY, Choi JW, Park YH, Kim YM, Yeo MK, Kim JM and Rha KS: Pattern of expression of cell cycle-related proteins in malignant transformation of sinonasal inverted papilloma. Am J Rhinol Allergy 25: 75-81, 2011.

23. Yoon AR, Gao R, Kaul Z, Choi IK, Ryu J, Noble JR, Kato Y, Saito S, Hirano T, Ishii T, et al: MicroRNA-296 is enriched in cancer cells and downregulates p21WAF1 mRNA expression via interaction with its 3' untranslated region. Nucleic Acids Res 39: 8078-8091, 2011

24. Würdinger T, Tannous BA, Saydam O, Skog J, Grau S, Soutschek J, Weissleder R, Breakefield XO and Krichevsky AM: miR-296 regulates growth factor receptor overexpression in angiogenic endothelial cells. Cancer Cell 14: 382-393, 2008.

25. Hong L, Han Y, Zhang H, Li M, Gong T, Sun L, Wu K, Zhao Q and Fan D: The prognostic and chemotherapeutic value of miR-296 in esophageal squamous cell carcinoma. Ann Surg 251: 1056-1063, 2010.

26. Liu X, Chen Q, Yan J, Wang Y, Zhu C, Chen C, Zhao X, Xu M, Sun Q, Deng R, et al: MiRNA-296-3p-ICAM-1 axis promotes metastasis of prostate cancer by possible enhancing survival of natural killer cell-resistant circulating tumour cells. Cell Death Dis 4: e928, 2013.

27. Chung CH, Parker JS, Karaca G, Wu J, Funkhouser WK, Moore D, Butterfoss D, Xiang D, Zanation A, Yin X, et al: Molecular classification of head and neck squamous cell carcinomas using patterns of gene expression. Cancer Cell 5: 489-500, 2004.

28. Hatakeyama H, Kondo T, Fujii K, Nakanishi Y, Kato H, Fukuda S and Hirohashi S: Protein clusters associated with carcinogenesis, histological differentiation and nodal metastasis in esophageal cancer. Proteomics 6: 6300-6316, 2006. 